\title{
Observation of spin-triplet superconductivity in Co-based Josephson Junctions
}

\author{
Trupti S. Khaire, Mazin A. Khasawneh, W. P. Pratt, Jr., Norman O. Birge* \\ Department of Physics and Astronomy, Michigan State University, East Lansing, Michigan 48824-2320, USA
}

(Dated: August 10, 2021)

\begin{abstract}
We have measured a long-range supercurrent in Josephson junctions containing Co (a strong ferromagnetic material) when we insert thin layers of either PdNi or CuNi weakly-ferromagnetic alloys between the Co and the two superconducting $\mathrm{Nb}$ electrodes. The critical current in such junctions hardly decays for Co thicknesses in the range of $12-28 \mathrm{~nm}$, whereas it decays very steeply in similar junctions without the alloy layers. The long-range supercurrent is controllable by the thickness of the alloy layer, reaching a maximum for a thickness of a few $\mathrm{nm}$. These experimental observations provide strong evidence for induced spin-triplet pair correlations, which have been predicted to occur in superconducting/ferromagnetic hybrid systems in the presence of certain types of magnetic inhomogeneity.
\end{abstract}

PACS numbers: 74.50.+r, 74.45.+c, 75.70.Cn, 74.20.Rp

When a conventional spin-singlet superconductor is brought into contact with a normal metal, superconducting pair correlations penetrate into the normal metal over distances as large as a micron at low temperature, creating the superconducting proximity effect [1]. If the normal metal is replaced by a ferromagnet, the pair correlations penetrate only a few nanometers, as the exchange field in the ferromagnet leads to a rapid loss of phase coherence between electrons with opposite-pointing spins [2, 3]. This limitation would not arise if the Cooper pairs in the superconductor had spin-triplet symmetry, which occurs only rarely in nature [4, 5]. It was predicted several years ago that spin-triplet superconducting correlations could be induced at the interface between a conventional spin-singlet superconductor and a ferromagnet with inhomogeneous magnetization [6, 7]. Moreover, these pair correlations are in a new symmetry class: they have even relative orbital angular momentum but are odd in frequency or time [8]. A promising hint of spin-triplet correlations in half-metallic $\mathrm{CrO}_{2}$ was reported in 2006 by Keizer et al. [9]; however there has been no confirmation of that result in the intervening time. Here we present strong evidence for spin-triplet pair correlations in Josephson junctions fabricated from common metals: $\mathrm{Nb}$ and $\mathrm{Co}$. The magnetic inhomogeneity is supplied by thin layers of a weakly-ferromagnetic alloy - either PdNi or $\mathrm{CuNi}$ - inserted between the $\mathrm{Co}$ and $\mathrm{Nb}$ layers. As the Co thickness is increased, the maximum supercurrent in the Josephson junctions decays very slowly - in sharp contrast to the very fast decay observed in similar junctions without these alloy layers [10]. The strength of the triplet correlations can be controlled by the thickness of the alloy layer, reaching its maximum for a thickness of a few $\mathrm{nm}$.

A schematic diagram of our Josephson junction samples is shown in Figure 1a. The entire multilayer structure up through the top Au layer is sputtered onto a Si substrate in a single run, without breaking vacuum between subsequent layers. The multilayers are subse- quently patterned into circular pillars using photolithography and Ar ion milling, after which the $\mathrm{SiO}_{x}$ insulating layer is thermally evaporated to isolate the top $\mathrm{Nb}$ contact from the base. Finally the top $\mathrm{Nb}$ contact is sputtered through a mechanical mask. The Au layer is fully superconducting due to the proximity effect with the surrounding $\mathrm{Nb}$ layers. The $\mathrm{Nb}$ superconducting layers have critical temperature near $9 \mathrm{~K}$, which allows us to measure the Josephson critical supercurrent at $4.2 \mathrm{~K}$ with the samples dipped in liquid helium. Details of our fabrication and measurement procedures are given in our previous publications [10, 11].
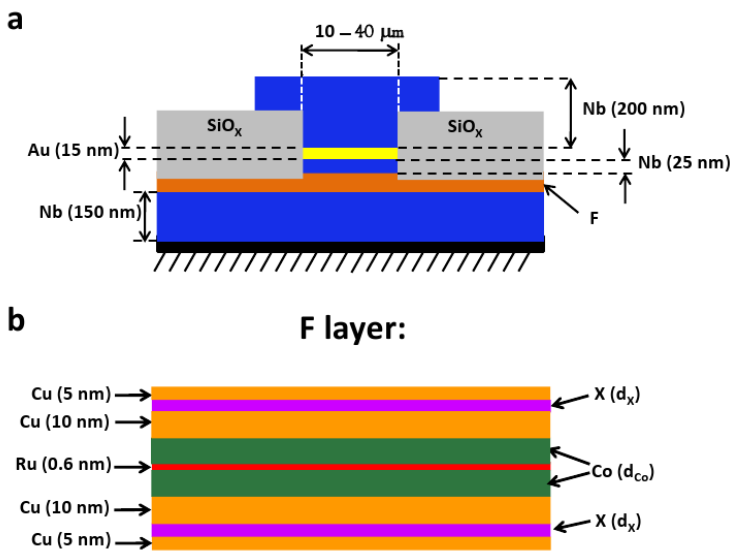

FIG. 1: (color online). a) Schematic diagram of the Josephson junction samples, shown in cross-section. b) Detailed sequence of the metal layers inside the Josephson junctions (labelled $\mathrm{F}$ in a). The layers labelled $\mathrm{X}$ are either $\mathrm{PdNi}$ or $\mathrm{CuNi}$ alloy. The functions of the various layers are described in the text. Only the thicknesses of the Co and X layers are varied in this work. The $\mathrm{Cu}$ buffer layers play no active role in the devices, but are important to isolate the $\mathrm{X}$ layers magnetically from the Co layers.

The detailed sequence of internal layers (labelled $\mathrm{F}$ for "ferromagnetic" in Figure 1a) is shown in Figure 1b. 
The layers labelled $\mathrm{X}$ represent either $\mathrm{Pd}_{0.88} \mathrm{Ni}_{0.12}$ or $\mathrm{Cu}_{0.48} \mathrm{Ni}_{0.52}$ ferromagnetic alloys. The purpose of the $\mathrm{Co}$ in the central $\mathrm{Co} / \mathrm{Ru} / \mathrm{Co}$ trilayer is to suppress the conventional spin-singlet Josephson supercurrent. The critical current of similar Josephson junctions containing $\mathrm{Co} / \mathrm{Ru} / \mathrm{Co}$ trilayers, but without the $\mathrm{X}$ layers, decays exponentially with increasing Co thickness with a decay constant of $2.34 \pm 0.08 \mathrm{~nm}$ [10]. The thin Ru layer induces antiparallel exchange coupling between the domains in the two Co layers [12], to produce nearly zero total magnetic flux in the junctions. As a result, the critical current vs. applied magnetic field data of these Josephson junctions exhibit nearly-ideal "Fraunhofer patterns," as shown in Figure 2. These patterns give us reliable measurements of the maximum possible critical current in each sample, while also indicating that the current flow in the junctions is uniform and that there are no shorts in the surrounding $\mathrm{SiO}$ insulator.

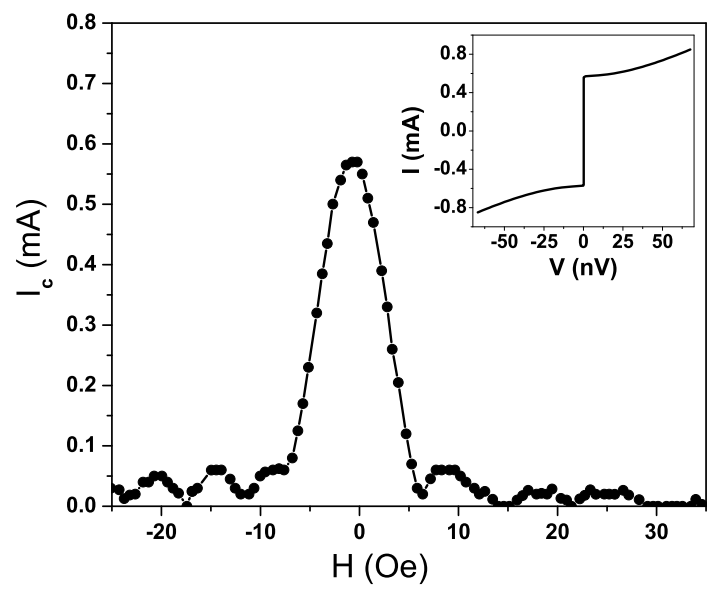

FIG. 2: Critical current $\left(I_{c}\right)$ vs. applied magnetic field $(H)$ for a $10 \mu \mathrm{m}$ diameter Josephson junction with $d_{C o}=13 \mathrm{~nm}$ and $d_{P d N i}=4 \mathrm{~nm}$, measured at $T=4.2 \mathrm{~K}$. The excellent "Fraunhofer pattern" results from cancellation of the intrinsic magnetic flux in the junction, due to antiparallel exchange coupling of the two Co layers via the thin $\mathrm{Ru}$ layer. (The lines are guides to the eye.) The inset shows the current-voltage $(I-V)$ characteristic of the junction at $H=0$.

We discuss first the case where $\mathrm{X}=\mathrm{Pd}_{0.88} \mathrm{Ni}_{0.12}$, a weakly-ferromagnetic alloy with a Curie temperature of $175 \mathrm{~K}$ [11]. Figure 3a shows the product of critical current and normal state resistance, $I_{c} R_{N}$, vs. total cobalt thickness, $D_{C o} \equiv 2 d_{C o}$, for a series of samples with fixed PdNi layer thickness, $d_{P d N i}=4 \mathrm{~nm}$. (The normal state resistance, $R_{N}$, is determined from the inverse slope of the $I-V$ curve for $I \gg I_{c}$.) There is no discernible decay of $I_{c} R_{N}$ for $D_{C o}>12 \mathrm{~nm}$. For comparison, Figure 3a also shows data from Ref. [10] for junctions not containing PdNi. In those samples $I_{c} R_{N}$ decays very rapidly with increasing $D_{C o}$. When $D_{C o}=20 \mathrm{~nm}, I_{c} R_{N}$ is over 100 times larger in the samples with PdNi than in the samples without PdNi. The long-range character of the Josephson current in samples with PdNi represents strong evidence for its spin-triplet nature.

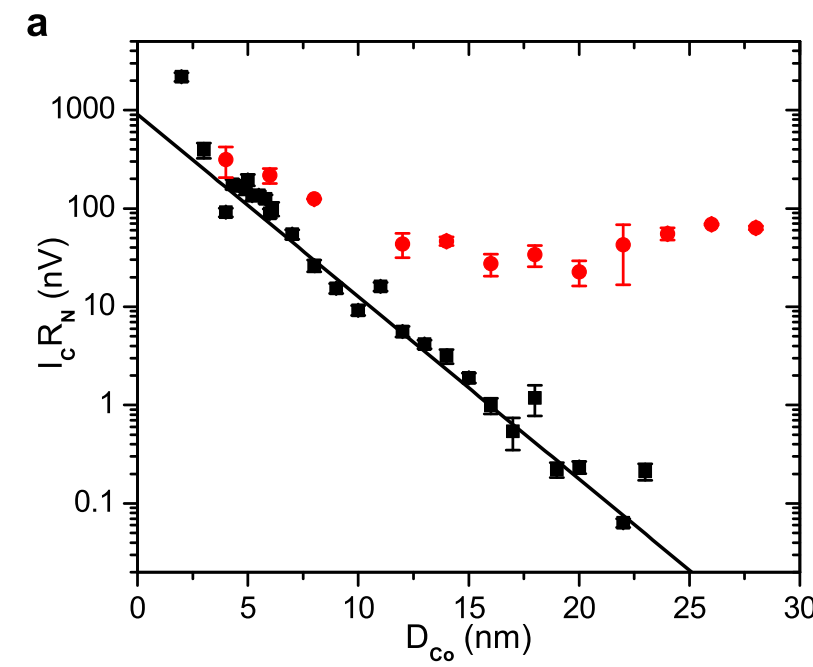

b

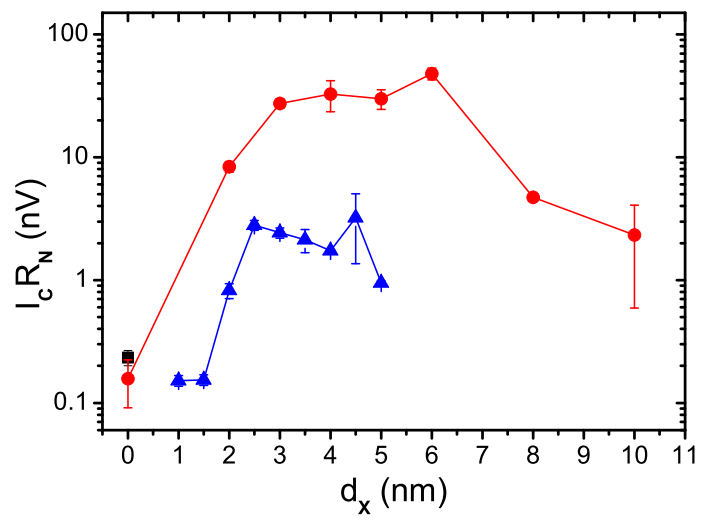

FIG. 3: (color online). a) Product of critical current times normal state resistance, $I_{c} R_{N}$, as a function of total Co thickness, $D_{C o}=2 d_{C o}$. Red circles represent junctions with $\mathrm{X}=$ $P d N i$ and $\mathrm{d}_{P d N i}=4 \mathrm{~nm}$, whereas black squares represent junctions with no X layer (taken from Ref. [10]). As $D_{C o}$ increases above $12 \mathrm{~nm}, I_{c} R_{N}$ hardly drops in samples with $\mathrm{PdNi}$, but drops very rapidly in samples without. (The solid line is a fit of the data without PdNi to a decaying exponential, also from Ref. [10].) Error bars represent the standard deviation determined from measurements of two or more pillars on the same substrate, or are set to $10 \%$ in the few cases where data from only a single pillar were available. $\mathbf{b},) I_{c} R_{N}$ product as a function of $d_{X}$ for two series of junctions with fixed $D_{C o}=20 \mathrm{~nm}$. Red circles: X $=$ PdNi; blue triangles: $\mathrm{X}=\mathrm{CuNi}$. (The square at $d_{X}=0$ is taken from Ref. [10].) In both cases, $I_{c} R_{N}$ first increases, then eventually decreases with increasing $d_{X}$. Lines are guides to the eye.

The subtle role of the $\mathrm{X}$ layers in enhancing the supercurrent is illustrated in Figure $3 \mathrm{~b}$, which shows $I_{c} R_{N}$ vs. $d_{X}$ with $\mathrm{X}=\mathrm{PdNi}$ or $\mathrm{CuNi}$ for two sets of samples with $D_{C o}$ fixed at $20 \mathrm{~nm}$. Without any $\mathrm{X}$ layer, $I_{c} R_{N}$ is very small, consistent with the data shown in 
Figure 3a. When the $\mathrm{X}$ layer reaches a critical thickness, $I_{c} R_{N}$ increases rapidly, indicating that spin-triplet pair correlations are being produced in the $\mathrm{Nb} / \mathrm{Cu} / \mathrm{X}$ interface region. $I_{c} R_{N}$ reaches a maximum for $d_{X}$ values of a few $\mathrm{nm}$, then decreases at larger values of $d_{X}$. We believe that the decrease in $I_{c} R_{N}$ at large $d_{X}$, visible for $\mathrm{X}=\mathrm{PdNi}$, is caused by the destruction of the spintriplet correlations created at the $\mathrm{Nb} / \mathrm{Cu} / \mathrm{X}$ interface due to spin memory loss in the bulk of the $\mathrm{X}$ layers. The spin memory lengths in these $\mathrm{PdNi}$ and $\mathrm{CuNi}$ alloys are very short - about $2.8 \mathrm{~nm}$ in $\mathrm{PdNi}$ [13] and $1.4 \mathrm{~nm}$ in $\mathrm{CuNi}$ [14]. This would explain why we found no evidence for spin-triplet supercurrent in our previous measurements of Josephson junctions containing only PdNi layers of thickness 30-100 nm 11. Evidently, a thin PdNi or CuNi layer is essential to produce spin-triplet correlations, whereas a thick layer suppresses them.

What essential properties give $\mathrm{PdNi}$ and $\mathrm{CuNi}$ their ability to produce spin-triplet pair correlations in these Josephson junctions? We speculate that the two crucial ingredients for generation of the triplet are domain size, which should be comparable to the superconducting coherence length in $\mathrm{Nb}$, and out-of-plane magnetocrystalline anisotropy. While the domain size of $\mathrm{PdNi}$ is not known, the domain size in $\mathrm{Cu}_{0.47} \mathrm{Ni}_{0.53}$ has recently been measured to be about $100 \mathrm{~nm}$ [15], which is not so different from the $\mathrm{Nb}$ coherence length $\xi_{S}=14 \mathrm{~nm}$. Competition between out-of-plane magnetocrystalline anisotropy and the in-plane shape anisotropy of thin films can lead to stripe domains with canted magnetization [16] and thus to non-collinear magnetizations in neighboring domains a key requirement for production of the triplet [8]. Both PdNi [11] and CuNi [17] are known to have out-of-plane magnetic anisotropy. In this context, it is interesting to note what happens when the $\mathrm{Cu}$ buffer layers between the $\mathrm{X}$ and Co layers are omitted. We have tried this for $\mathrm{X}=\mathrm{PdNi}$, and found that the supercurrent is much smaller than in samples with $\mathrm{Cu}$ buffer layers. Presumably the domain structure of the $\mathrm{PdNi}$ is changed by exchange-coupling to the $\mathrm{Co}$, in a way that is detrimental to production of the triplet correlations. We suspect that the PdNi magnetization is forced to lie in-plane near the interface with Co, which leads to less non-collinear magnetization in neighboring domains 13 .

It is natural to ask whether there are other materials besides $\mathrm{PdNi}$ or $\mathrm{CuNi}$ that can produce spin-triplet correlations. Clearly Co alone is not sufficient, as demonstrated by the samples without X layers [10]. Scanning electron microscopy with polarization analysis (SEMPA) measurements on Co films grown under similar conditions as ours reveal magnetic domains with typical sizes of a few microns, but with the magnetization directions of neighboring domains largely antiparallel [18]. Noncollinear magnetization resides only in the domain walls, which is apparently not enough to produce a significant amount of spin-triplet. Aside from the presence of non- collinear magnetizations, theory suggests that any "spinactive" interface between a superconductor and a ferromagnet can produce spin-triplet correlations 19]. We have tried using $\mathrm{X}=\mathrm{Cu}_{0.94} \mathrm{Pt}_{0.06}$, an alloy with strong spin-orbit scattering, but preliminary data show very little, if any, signature of the triplet. We predict that PdCo, another weakly ferromagnetic alloy with properties similar to those of $\mathrm{PdNi}$, will produce triplet correlations.

Comparison of our results with theory is problematic. The magnitude of the spin-triplet supercurrent depends on the details of the PdNi or CuNi domain structure, while theoretical calculations exist only for idealized magnetic configurations. More useful is a discussion of the decay lengths of the spin-singlet and spin-triplet supercurrents. In the "dirty" limit, where the mean free path, $l_{e}$, is the shortest relevant length scale in the problem, the spin-singlet supercurrent should decay on the length scale $\xi_{F}=\sqrt{\hbar D_{F} / E_{e x}}$, where $D_{F}$ and $E_{e x}$ are the electron diffusion constant and exchange energy in the ferromagnet. Josephson junctions containing Co, however, are in the "intermediate" limit, with $l_{e}$ longer than $\xi_{F}$, but shorter than $\xi_{S}$, the superconducting coherence length. In that limit, the spin-singlet supercurrent decays on the length scale $l_{e}$, which is estimated to be $2.4-3.0$ $\mathrm{nm}$ from previous studies [10, 20]. Spin-triplet supercurrent, in contrast, should decay over a much longer length scale given by the smaller of the normal metal coherence length, $\xi_{N}=\sqrt{\hbar D_{F} / 2 \pi k_{B} T}$, or the spin memory length, $L_{s f}=\sqrt{\hbar D_{F} / \tau_{s f}}$, where $\tau_{s f}$ is the mean time between spin-flip or spin-orbit scattering events. Estimation of $D_{F}$ for Co is difficult due to its strong ferromagnetism and to the widely-varying densities of states and Fermi velocities of the different bands. From our measured Co resistivity, the Einstein relation, and the densities of states of majority and minority electrons at the Fermi surface [21], we estimate $D_{F}=5 \times 10^{-3} \mathrm{~m}^{2} / \mathrm{s}$ and $5 \times 10^{-4} \mathrm{~m}^{2} / \mathrm{s}$ for the majority and minority electrons, respectively, which give $\xi_{N}=40 \mathrm{~nm}$ and $10 \mathrm{~nm}$ at $T=4.2 \mathrm{~K} . L_{s f}$ in Co has been measured to be about 60 $\mathrm{nm}$, also with large uncertainty [22, 23]. Unfortunately, sample-to-sample fluctuations in the experimental data in Figure 3a mask any discernible decay for $D_{C o}$ between 12 and $28 \mathrm{~nm}$. Better statistics or data over a much larger range of $D_{C o}$ will be needed to extract a meaningful estimate of the decay length for the spin-triplet supercurrent. We have not yet attempted to increase $D_{C o}$ much more due to concerns about the efficacy of the $\mathrm{Co} / \mathrm{Ru} / \mathrm{Co}$ exchange-coupled trilayer.

The spin-triplet pair correlations observed here and discussed in Ref. 8] are quite different from those believed to occur in materials such as $\mathrm{Sr}_{2} \mathrm{RuO}_{4}$ [4]. The Cooper pairs in the latter satisfy the Spin-Statistics Theorem of quantum mechanics by having odd relative orbital angular momentum (p-wave). According to theory [8], the triplet pair correlations induced in superconductor/ferromagnet hybrid systems have even relative or- 
bital angular momentum; in particular, they can be swave, which implies that they are robust in the presence of disorder. Quantum mechanics is not violated because the correlations are odd in frequency, or equivalently odd under time reversal. This idea, first proposed in a model for liquid helium-3 by Berezinskii [24], is counterintuitive, as it implies that the equal-time pair correlation function vanishes. Theoretical guidance is needed to find a direct method to probe the odd-frequency aspect of the pair correlations experimentally, rather than relying on the observed long-range supercurrent to deduce the spintriplet character combined with robustness to disorder.

Looking back, there were hints of long-range proximity effects in superconducting/ferromagnetic hybrid systems as early as 10 years ago 25, 26, 27, 28], but there was no way to control the observed effects. More recently, Sosnin et al. [29] observed phase-coherent oscillations in the resistance of a Ho wire connected to two superconductors, but the authors did not observe a Josephson supercurrent, nor did they comment on its absence. The observation by Keizer et al. [9] of a supercurrent through $\mathrm{CrO}_{2}$ was an exciting advance, but the critical currents in those samples varied by two orders of magnitude in similar samples. We anticipate that our results, which exhibit systematic dependence of the spin-triplet supercurrent on $\mathrm{PdNi}$ or $\mathrm{CuNi}$ thickness, will pave the way to many new experiments [30, 31].

Acknowledgments: We thank S. Bergeret, P. Brouwer, K.B. Evetov, M. Stiles, and A. Volkov for helpful discussions, R. Loloee, B. Bi, and Y. Wang for technical assistance, and use of the W.M. Keck Microfabrication Facility. This work was supported by the U.S. Department of Energy under grant DE-FG02-06ER46341.

* Electronic address: birge@pa.msu.edu

[1] G. Deutscher and P.G. de Gennes, in Superconductivity, edited by R.G. Parks (Dekker, New York, 1969), pp. 1005.

[2] A. Buzdin, L.N. Bulaevskii, and S.V. Panyukov, JETP Lett. 35, 178 (1982).

[3] E.A. Demler, G.B. Arnold, and M.R. Beasley, Phys. Rev. B 55, 15174 (1997).

[4] A.P. Mackenzie, and Y. Maeno, Rev. Mod. Phys. 75, 657 (2003).
[5] S.S. Saxena et al. Nature 406, 587 (2000).

[6] F.S. Bergeret, A.F. Volkov, and K.B. Efetov, Phys. Rev. Lett. 86, 4096 (2001).

[7] A. Kadigrobov, R.I. Shekhter, and M. Jonson, Europhys. Lett. 54(3), 394 (2001).

[8] F.S. Bergeret, A.F. Volkov, and K.B. Efetov, Rev. Mod. Phys. 77, 1321 (2005).

[9] R.S. Keizer, S.T.B. Goennenwein, T.M. Klapwijk, G. Miao, G. Xiao, and A. Gupta, Nature (London) 439, 825 (2006).

[10] M.A. Khasawneh, W.P. Pratt, and N.O. Birge, Phys. Rev. B 80, 020506(R) (2009).

[11] T.S. Khaire, W.P. Pratt, Jr., and N.O. Birge, Phys. Rev. B 79, 094523 (2009).

[12] S.S.P. Parkin, N. More, and K.P. Roche, Phys. Rev. Lett. 64, 2304 (1990).

[13] H. Arham, T.S. Khaire, R. Loloee, W.P. Pratt, and N.O. Birge, Phys. Rev. B 80, 174515 (2009).

[14] V. A. Oboznov, V. V. Bol'ginov, A. K. Feofanov, V. V. Ryazanov, and A.I. Buzdin, Phys. Rev. Lett. 96, 197003 (2006).

[15] I.S. Veshchunov, et al. JETP Lett. 88, 758 (2009).

[16] T. Koikeda, K. Suzuki, and S. Chikazumi, Appl. Phys. Lett. 4, 160 (1964).

[17] A. Ruotolo, C. Bell, C.W. Leung, and M.G. Blamire, J. Appl. Phys. 96, 512 (2004).

[18] J.A. Borchers, et al. Phys. Rev. Lett. 82, 2796 (1999).

[19] M. Eschrig, J. Kopu, J.C. Cuevas, and G. Schön, Phys. Rev. Lett. 90, 137003 (2003).

[20] J.W.A. Robinson, S. Piano, G. Burnell, C. Bell and M.G. Blamire, Phys. Rev. Lett. 97, 177003 (2006).

[21] D.A. Papaconstantopoulos, Handbook of the band structure of elemental solids (Plenum Press, New York, 1986).

[22] L. Piraux, S. Dubois, A. Fert, and L. Belliard, Euro. Phys. J. B 4, 413 (1998).

[23] J. Bass, and W.P. Pratt, J. Phys.: Condens. Matter 19, 183201 (2007).

[24] V.L. Berezinskii, JETP Lett. 20, 287 (1974).

[25] M. Giroud, H. Courtois, K. Hasselbach, D. Mailly, and B. Pannetier, Phys. Rev. B 58, R11872 (1998).

[26] M.D. Lawrence, and H. Giordano, J. Phys.: Condens. Matter 11, 1089 (1999).

[27] V. T. Petrashov, I.A. Sosnin, I. Cox, A. Parsons, and C. Troadec, Phys. Rev. Lett. 83, 3281 (1999).

[28] V. Pena, et al. Phys. Rev. B 69, 224502 (2004).

[29] I. Sosnin, H. Cho, V.T. Petrashov, and A.F. Volkov, Phys. Rev. Lett. 96, 157002 (2006).

[30] T. Kontos, M. Aprili, J. Lesueur, and X. Grison, Phys. Rev. Lett. 86, 304 (2001).

[31] P. SanGiorgio, S. Reymond, M.R. Beasley, J.H. Kwon, and K. Char, Phys. Rev. Lett. 100, 237002 (2008). 